\title{
Pacific
}

Journal of

Mathematics

\section{FORMAL EQUIVALENCE OF POISSON STRUCTURES} AROUND POISSON SUBMANIFOLDS

\author{
IOAN MĂRCUȚ
}

Volume $255 \quad$ No. 2

February 2012 


\title{
FORMAL EQUIVALENCE OF POISSON STRUCTURES AROUND POISSON SUBMANIFOLDS
}

\author{
IOAN MĂRCUT,
}

\begin{abstract}
Let $(M, \pi)$ be a Poisson manifold. A Poisson submanifold $P \subset M$ gives rise to a Lie algebroid $A_{P} \rightarrow P$. Formal deformations of $\pi$ around $P$ are controlled by certain cohomology groups associated to $A_{P}$. Assuming that these groups vanish, we prove that $\pi$ is formally rigid around $P$; that is, any other Poisson structure on $M$, with the same first-order jet along $P$, is formally Poisson diffeomorphic to $\pi$. When $P$ is a symplectic leaf, we find a list of criteria that are sufficient for these cohomological obstructions to vanish. In particular, we obtain a formal version of the normal form theorem for Poisson manifolds around symplectic leaves.
\end{abstract}

\section{Introduction}

A Poisson bracket on a manifold $M$ is a Poisson algebra structure on the space of smooth functions on $M$, that is, a Lie bracket $\{\cdot, \cdot\}$ on $C^{\infty}(M)$ satisfying the derivation property

$$
\{f, g h\}=\{f, g\} h+\{f, h\} g \text { for all } f, g, h \in C^{\infty}(M) .
$$

Equivalently, it can be given by a bivector $\pi \in \mathfrak{X}^{2}(M)$ that satisfies $[\pi, \pi]=0$. The two definitions are related by the formula

$$
\langle\pi, d f \wedge d g\rangle=\{f, g\} \text { for all } f, g \in C^{\infty}(M) .
$$

An immersed submanifold $\iota: P \rightarrow M$ is called a Poisson submanifold of $M$ if $\pi$ is tangent to $P$. This ensures that $\pi_{\mid P}$ is a Poisson structure on $P$ for which the restriction map

$$
\iota^{*}: C^{\infty}(M) \rightarrow C^{\infty}(P)
$$

is a Lie algebra homomorphism. We regard the Poisson algebra $\left(C^{\infty}(P),\{\cdot, \cdot\}\right)$ as the 0th-order approximation of the Poisson structure on $M$. If $P$ is embedded,

This research was supported by the NWO Vidi Project "Poisson topology."

MSC2010: primary 53D17, 58H15; secondary 70K45, 17B55, $17 \mathrm{~B} 70$.

Keywords: Poisson geometry, Lie algebroid, graded Lie algebra. 
then $P$ is a Poisson submanifold if and only if its vanishing ideal

$$
I(P)=\left\{f \in C^{\infty}(M) \mid \iota^{*}(f)=0\right\}
$$

is an ideal in the Lie algebra $\left(C^{\infty}(M),\{\cdot, \cdot\}\right)$. Assuming that $P$ is also closed ${ }^{1}$, we have a canonical identification of Poisson algebras

$$
\left(C^{\infty}(P),\{\cdot, \cdot\}\right)=\left(C^{\infty}(M) / I(P),\{\cdot, \cdot\}\right) .
$$

This gives a recipe for constructing higher-order approximations. For example, the first-order approximation fits into an exact sequence of Poisson algebras

$$
\begin{aligned}
0 \rightarrow\left(I(P) / I^{2}(P),\{\cdot, \cdot\}\right) & \\
& \rightarrow\left(C^{\infty}(M) / I^{2}(P),\{\cdot, \cdot\}\right) \rightarrow\left(C^{\infty}(P),\{\cdot, \cdot\}\right) \rightarrow 0 .
\end{aligned}
$$

The Poisson algebra structures in this sequence depend only on $j_{\mid P}^{1} \pi$, the first jet of $\pi$ along $P$. A better way to describe (2) is using the language of Lie algebroids. As explained in Section 2, the extension (2) gives rise to a Lie algebroid structure $A_{P}$ on $T_{P}^{*} M$ that fits into a short exact sequence of Lie algebroids

$$
0 \rightarrow T P^{\circ} \rightarrow A_{P} \rightarrow T^{*} P \rightarrow 0,
$$

where $T P^{\circ} \subset T_{P}^{*} M=A_{P}$ is the annihilator of $T P$ and $T^{*} P$ is the cotangent Lie algebroid of $(P,\{\cdot, \cdot\})$. In particular, we obtain a representation of $A_{P}$ on $T P^{\circ}$, and thus also on its symmetric powers $\mathscr{S}^{k}\left(T P^{\circ}\right)$.

We study formal rigidity of Poisson structures around Poisson submanifolds. In general, deformation and rigidity problems in Poisson geometry are controlled by the Poisson cohomology groups $H_{\pi}^{\bullet}(M)$, which are the cohomology of the complex of multivector fields $\left(\mathfrak{X}^{\bullet}(M), d_{\pi}\right)$, where

$$
d_{\pi}:=[\pi, \cdot] .
$$

For a Poisson submanifold $P$, this $d_{\pi}$ induces a differential on $\mathfrak{X}^{\bullet}(M)_{\mid P}$, the complex of multivector fields along $M$. The corresponding cohomology, denoted by $H_{\pi}^{\bullet}(M, P)$, is called the Poisson cohomology relative to $P$ [Ginzburg and $\mathrm{Lu}$ 1992]. The formal rigidity of Poisson structures around Poisson submanifolds is controlled by a version of this cohomology with coefficients. Lie algebroids provide the right setting to make this precise; that is, the relative Poisson cohomology groups can be computed as the cohomology of the Lie algebroid $A_{P}$

$$
H_{\pi}^{\bullet}(M, P)=H^{\bullet}\left(A_{P}\right)
$$

${ }^{1}$ Since we study local properties of $(M, \pi)$ around $P$, only the condition that $P$ is embedded is essential; closeness can be achieved by replacing $M$ with a tubular neighborhood of $P$. 
and the cohomology groups of $A_{P}$ with coefficients in $\varphi^{k}\left(T P^{\circ}\right)$, which we denote by $H^{\bullet}\left(A_{P} ; \mathscr{S}^{k}\left(T P^{\circ}\right)\right.$ ), control formal rigidity (see Section 2 for the definition of Lie algebroid cohomology).

Our main result is the following:

Theorem 1.1. Let $\pi_{1}$ and $\pi_{2}$ be two Poisson structures on $M$, such that $P \subset M$ is an embedded Poisson submanifold for both, and such that they have the same first-order jet along $P$. If their common algebroid $A_{P}$ has the property that

$$
H^{2}\left(A_{P} ; \mathscr{Y}^{k}\left(T P^{\circ}\right)\right)=0 \text { for all } k \geq 2,
$$

then the two structures are formally Poisson diffeomorphic. More precisely, there exists a diffeomorphism

$$
\psi: \mathscr{U} \rightarrow \mathscr{V}
$$

with $d \psi_{\mid T_{P} M}=\mathrm{id}_{T_{P} M}$, where $\mathcal{U}$ and $\mathscr{V}$ are open neighborhoods of $P$, such that $\pi_{1 \mid u}$ and $\psi^{*}\left(\pi_{2 \mid v}\right)$ have the same infinite jet along $P$ :

$$
j_{\mid P}^{\infty}\left(\pi_{1 \mid U}\right)=j_{\mid P}^{\infty}\left(\psi^{*}\left(\pi_{2 \mid V}\right)\right) .
$$

Applying Theorem 1.1 to the linear Poisson structure on the dual of a compact, semisimple Lie algebra, we obtain the following result.

Corollary 1.2. Let $\mathfrak{g}$ be a semisimple Lie algebra of compact type and consider $\pi_{\text {lin }}$ the linear Poisson structure on $\mathfrak{g}^{*}$. Let $\mathfrak{S}(\mathfrak{g}) \subset \mathfrak{g}^{*}$ be the sphere in $\mathfrak{g}^{*}$ centered at 0 , of radius 1 with respect to some invariant inner product. Then $\mathbb{S}(\mathfrak{g})$ is a Poisson submanifold, and any Poisson structure $\pi_{1}$ defined in some open neighborhood of $\mathbb{S}(\mathfrak{g})$, such that

$$
j_{\mid S(\mathfrak{g})}^{1}\left(\pi_{\operatorname{lin}}\right)=j_{\mid \mathbb{S}(\mathfrak{g})}^{1}\left(\pi_{1}\right),
$$

is formally Poisson diffeomorphic to $\pi_{\mathrm{lin}}$.

The symplectic leaves of $(M, \pi)$ are Poisson submanifolds of a special type. Recall that a Poisson manifold carries a canonical singular foliation whose leaves are the maximal integral submanifolds of the distribution $\pi^{\sharp}\left(T^{*} M\right)$. Such a leaf $S$ has a natural symplectic structure given by $\omega_{S}:=\pi_{\mid S}^{-1}$. If $\left(S, \omega_{S}\right) \subset(M, \pi)$ is an embedded symplectic leaf, then the Lie algebroid extension (3) - which encodes only the first-order jet $\pi$ along $S$ - can be used to construct a second Poisson structure $\pi_{S}^{1}$, called the first-order approximation of $\pi$ around $S$, defined on some open neighborhood of $S$ and having the same first jet as $\pi$ along $S$.

In [Crainic and Mărcuţ 2010] we obtained a normal form theorem for Poisson structures around symplectic leaves: we proved that, under some assumptions on the first jet of $\pi$ along $S$, the Poisson structures $\pi$ and $\pi_{S}^{1}$ are Poisson diffeomorphic around $S$. Our goal is to give a formal version of this result, which we state below in its most general form (observe that it is a direct consequence of Theorem 1.1). 
Theorem 1.3. Let $(M, \pi)$ be a Poisson manifold and $S \subset M$ an embedded symplectic leaf. If the cohomology groups

$$
H^{2}\left(A_{S}, \varphi^{k}\left(T S^{\circ}\right)\right)
$$

vanish for all $k \geq 2$, then $\pi$ is formally Poisson diffeomorphic to its first-order approximation around $S$.

In many cases we show that these cohomological obstructions vanish, and we obtain the following corollaries.

Corollary 1.4. Let $(M, \pi)$ be a Poisson manifold and $S \subset M$ an embedded symplectic leaf. Assume that the Poisson homotopy cover of $S$ is a smooth principal bundle with vanishing second de Rham cohomology group, and that its structure group $G$ satisfies

$$
H_{\text {diff }}^{2}\left(G, \mathscr{Y}^{k}(\mathfrak{g})\right)=0 \quad \text { for all } k \geq 2,
$$

where $\mathfrak{g}$ is the Lie algebra of $G$, and $H_{\text {diff }}^{\bullet}\left(G, \varphi^{k}(\mathfrak{g})\right)$ denotes the differentiable cohomology of $G$ with coefficients in the $k$-th symmetric power of the adjoint representation. Then $\pi$ is formally Poisson diffeomorphic to its first-order approximation around $S$.

Since the differentiable cohomology of compact groups vanishes, we obtain the following immediate corollary.

Corollary 1.5. Let $(M, \pi)$ be a Poisson manifold and $S \subset M$ an embedded symplectic leaf. If the Poisson homotopy cover of $S$ is a smooth principal bundle with vanishing second de Rham cohomology group and compact structure group, then $\pi$ is formally Poisson diffeomorphic to its first-order approximation around $S$.

The next consequence is bit more technical:

Corollary 1.6. Let $(M, \pi)$ be a Poisson manifold and $S \subset M$ an embedded symplectic leaf whose isotropy Lie algebra is reductive. If the abelianization algebroid

$$
A_{S}^{\mathrm{ab}}:=A_{S} /\left[T S^{\circ}, T S^{\circ}\right]
$$

is integrable by a simply connected principal bundle with compact structure group and vanishing second de Rham cohomology group, then $\pi$ is formally Poisson diffeomorphic to its first-order approximation around $S$.

Corollary 1.7. Let $(M, \pi)$ be a Poisson manifold and $S \subset M$ an embedded symplectic leaf through $x \in M$. If the isotropy Lie algebra at $x$ is semisimple, $\pi_{1}(S, x)$ is finite, and $\pi_{2}(S, x)$ is torsion, then $\pi$ is formally Poisson diffeomorphic to its first-order approximation around $S$. 
Some related results. The first-order approximation of a Poisson manifold $(M, \pi)$ around a one-point leaf $x$ (a zero of $\pi$ ) is the linear Lie-Poisson structure on $\mathfrak{g}_{x}^{*}$, the dual of the isotropy Lie algebra at $x$. Formal linearization in this setup was proven by Weinstein [1983] for semisimple $\mathfrak{g}_{x}$. This case is also covered by our Corollary 1.7. Under the stronger assumption that $\mathfrak{g}_{x}$ is semisimple of compact type, Conn [1985] proved that a neighborhood of $x$ is in fact Poisson diffeomorphic to an open neighborhood of 0 in the local model $\mathfrak{g}_{x}^{*}$.

Vorobjev [2001] constructed the first-order approximation around arbitrary symplectic leaves (see [Crainic and Mărcuţ 2010] for a more geometrical approach).

A weaker version of our Theorem 1.1 - of which we became aware only at the end of this research — was stated by Itskov et al. [1998]. They work around compact symplectic leaves instead of embedded Poisson submanifolds, proving that for each $k$, there exists a diffeomorphism that identifies the Poisson structures up to order $k$ [Itskov et al. 1998, Theorem 7.1]. Compactness of the leaf is nevertheless too strong an assumption for formal equivalence. For example, they conclude in their Corollary 7.4 that hypotheses similar to those in our Corollary 1.7 imply the vanishing of the cohomology groups $H^{2}\left(A_{S}, \varphi^{k}\left(T S^{\circ}\right)\right)$, but also remark that compactness of the leaf is incompatible with these assumptions (it forces $S$ to be a point).

To prove Theorem 1.1, we reduce it to a result on the equivalence of MaurerCartan elements in complete graded Lie algebras, which we prove in the Appendix. The same criteria for equivalence of Maurer-Cartan elements, but in the context of differential graded algebras, can be found in [Abad et al. 2010, Appendix A].

To prove the vanishing of the cohomological obstructions, and the corollaries listed above, we use techniques such as Whitehead's Lemma for semisimple Lie algebras and spectral sequences for Lie algebroids, but also the more powerful techniques developed in [Crainic 2003], such as the Van Est map and vanishing of cohomology of proper groupoids.

Theorem [Crainic and Mărcuţ 2010, main result]. Let $(M, \pi)$ be a Poisson manifold and $S \subset M$ an embedded symplectic leaf; $\pi$ is Poisson diffeomorphic to its first-order approximation around $S$ if the following conditions are satisfied:

- the Poisson homotopy cover P of $S$ is smooth;

- $H_{\mathrm{dR}}^{2}(P)=0$;

- the structure group of $P$ is compact;

- $S$ is compact.

The first three conditions are the hypotheses of Corollary 1.5. So, giving up on compactness of the leaf, we still conclude that $\pi$ and its first-order approximation are formally Poisson diffeomorphic. Nevertheless, the conditions of Corollary 1.5 
are too strong in the formal setting; they force the semisimple part of the isotropy Lie algebra to be compact. Thus we consider the more technical Corollary 1.6 to be the correct analog in the formal category of the normal form theorem in [Crainic and Mărcuţ 2010]. In fact, Corollary 1.5 is a consequence of Corollary 1.6; it is precisely the case when the semisimple part of the isotropy Lie algebra is compact.

\section{The first-order data}

We recall some definitions; for more on Lie algebroids, see [Mackenzie 1987].

Definitions 2.1. A Lie algebroid over a manifold $B$ is a vector bundle $A \rightarrow B$ endowed with a Lie bracket $[\cdot, \cdot]$ on its space of sections $\Gamma(\mathscr{A})$ and a vector bundle map $\rho: \mathscr{A} \rightarrow T B$, called the anchor, which satisfy the Leibniz identity:

$$
[\alpha, f \beta]=f[\alpha, \beta]+L_{\rho(\alpha)}(f) \beta \quad \text { for all } f \in C^{\infty}(B), \alpha, \beta \in \Gamma(\mathscr{A}) .
$$

A representation of $\mathscr{A}$ is a vector bundle $E \rightarrow B$ endowed with a bilinear map

$$
\nabla: \Gamma(\mathscr{A}) \times \Gamma(E) \rightarrow \Gamma(E)
$$

satisfying

$$
\nabla_{f \alpha}(s)=f \nabla_{\alpha}(s), \quad \nabla_{\alpha}(f s)=f \nabla_{\alpha}(s)+L_{\rho(\alpha)}(f) s,
$$

and the flatness condition

$$
\nabla_{\alpha} \nabla_{\beta}(s)-\nabla_{\beta} \nabla_{\alpha}(s)=\nabla_{[\alpha, \beta]}(s) .
$$

The cohomology of a Lie algebroid $(\mathscr{A},[\cdot, \cdot], \rho)$ with coefficients in a representation $(E, \nabla)$ is defined by the complex $\Omega^{\bullet}(\mathscr{A}, E):=\Gamma\left(\Lambda^{\bullet} \mathscr{A}^{*} \otimes E\right)$ with differential given by the classical Koszul formula:

$$
\begin{aligned}
d_{\nabla} \omega\left(\alpha_{0}, \ldots, \alpha_{q}\right)= & \sum_{i}(-1)^{i} \nabla_{\alpha_{i}}\left(\omega\left(\alpha_{1}, \ldots, \widehat{\alpha}_{i}, \ldots, \alpha_{q}\right)\right) \\
& +\sum_{i<j}(-1)^{i+j} \omega\left(\left[\alpha_{i}, \alpha_{j}\right], \ldots, \widehat{\alpha}_{i}, \ldots, \widehat{\alpha}_{j}, \ldots, \alpha_{q}\right) .
\end{aligned}
$$

The corresponding cohomology groups are denoted by $H^{\bullet}(\mathscr{A}, E)$.

To a Poisson manifold $(M, \pi)$ one can associate a Lie algebroid structure on the cotangent bundle $T^{*} M$, with anchor given by $\pi$ viewed as a bundle map

$$
\pi^{\sharp}: T^{*} M \rightarrow T M
$$

and bracket uniquely determined by

$$
[d f, d g]:=d\{f, g\} \quad \text { for all } f, g \in C^{\infty}(M) ;
$$

see [Vaisman 1994] for details. 
Let $P \subset M$ be an embedded Poisson submanifold. Since $\pi$ is tangent to $P$, it is easy to see that the algebroid structure can be restricted to $P$, in the sense that there is a unique Lie algebroid structure on $A_{P}:=T_{P}^{*} M$ with anchor $\pi_{\mid P}^{\sharp}$ and bracket such that the restriction map $\Gamma\left(T^{*} M\right) \rightarrow \Gamma\left(A_{P}\right)$ is a Lie algebra homomorphism. The dual of the inclusion $T P \subset T_{P} M$ gives a map $A_{P} \rightarrow T^{*} P$ that is a Lie algebroid homomorphism, where $T^{*} P$ is the cotangent Lie algebroid of $\left(P, \pi_{\mid P}\right)$. This way we obtain the extension of Lie algebroids from the introduction:

$$
0 \rightarrow\left(T P^{\circ},[\cdot, \cdot]\right) \rightarrow\left(A_{P},[\cdot, \cdot]\right) \rightarrow\left(T^{*} P,[\cdot, \cdot]\right) \rightarrow 0 .
$$

This short exact sequence implies that $T P^{\circ}$ is an ideal in $\left(A_{P},[\cdot, \cdot]\right)$; therefore

$$
\nabla: \Gamma\left(A_{P}\right) \times \Gamma\left(T P^{\circ}\right) \rightarrow \Gamma\left(T P^{\circ}\right), \quad \nabla_{\alpha}(\eta):=[\alpha, \eta]
$$

defines a representation of $A_{P}$ on $T P^{\circ}$, and thus on its symmetric powers $\mathscr{S}^{k}\left(T P^{\circ}\right)$. The resulting cohomology groups are the obstructions appearing in Theorems 1.1 and 1.3. The Lie algebroid structures on $A_{P}$ and the sequence (4) depend only on the first jet of $\pi$ along $P$ (that is, the brackets and anchors can be expressed in terms of $\pi_{\mid P}$ and the first-order derivatives of $\pi$ restricted to $P$ ).

Remark 2.2. We regard the Lie algebroid $A_{P}$ as the first-order approximation of the Poisson bracket at $P$. To justify this interpretation, fix a Poisson structure $\pi_{P}$ on $P$, where $P \subset M$ is a closed embedded submanifold. Then there is a one-to-one correspondence between Poisson algebra structures on the commutative algebra $C^{\infty}(M) / I^{2}(P)$, which fit into the short exact sequence

$$
\begin{aligned}
0 \rightarrow\left(I(P) / I^{2}(P),\{\cdot, \cdot\}\right) & \\
& \rightarrow\left(C^{\infty}(M) / I^{2}(P),\{\cdot, \cdot\}\right) \rightarrow\left(C^{\infty}(P),\{\cdot, \cdot\}\right) \rightarrow 0,
\end{aligned}
$$

and Lie algebroid structures on $A_{P}:=T_{P}^{*} M$, which fit into a sequence of the form

$$
0 \rightarrow\left(T P^{\circ},[\cdot, \cdot]\right) \rightarrow\left(A_{P},[\cdot, \cdot]\right) \rightarrow\left(T^{*} P,[\cdot, \cdot]\right) \rightarrow 0 .
$$

The exterior derivative induces a map

$$
d: C^{\infty}(M) / I^{2}(P) \rightarrow \Gamma\left(A_{P}\right),
$$

and the correspondence between the brackets is uniquely determined by the fact that this is a Lie algebra homomorphism.

Example 2.3. Consider $P:=\mathbb{R}^{2}$ as the submanifold $\{z=0\} \subset M:=\mathbb{R}^{3}$. We construct a first-order extension of the trivial Poisson structure on $P$ to $M$, that is, a Poisson algebra structure on the commutative algebra

$$
C^{\infty}(M) / I^{2}(P)=C^{\infty}(M) /\left(z^{2}\right)=C^{\infty}(P) \oplus z C^{\infty}(P)
$$


with the property that $\{f, g\} \in(z)$, for all $f, g \in C^{\infty}(M) /\left(z^{2}\right)$. Explicitly, define

$$
\{f, g\}=z\left(\frac{\partial f}{\partial x} \frac{\partial g}{\partial y}-\frac{\partial f}{\partial y} \frac{\partial g}{\partial x}+x \frac{\partial f}{\partial x} \frac{\partial g}{\partial z}-x \frac{\partial f}{\partial z} \frac{\partial g}{\partial x}\right) \bmod \left(z^{2}\right) .
$$

A straightforward computation yields that $\{\cdot, \cdot\}$ satisfies the Jacobi identity, and therefore we have an extension of Poisson algebras

$$
0 \rightarrow z C^{\infty}(P) \rightarrow C^{\infty}(P) \oplus z C^{\infty}(P) \rightarrow C^{\infty}(P) \rightarrow 0,
$$

where the Poisson bracket on $P$ is zero. The total space of the corresponding Lie algebroid $A_{P}$ is $\mathbb{R}^{3} \times P \rightarrow P$. The bracket is given on the global frame $d x_{\mid P}, d y_{\mid P}$, $d z_{\mid P}$ by

$$
\left[d x_{\mid P}, d y_{\mid P}\right]=d z_{\mid P}, \quad\left[d y_{\mid P}, d z_{\mid P}\right]=0, \quad\left[d x_{\mid P}, d z_{\mid P}\right]=x d z_{\mid P},
$$

and extended bilinearly to all sections, since the anchor is zero.

Nevertheless, there is no Poisson structure on $M$ (nor on any open neighborhood of $P$ ) that has this Poisson algebra as its first-order approximation. Assume, to the contrary, that on some open neighborhood $U$ of $P$ such a Poisson structure exists. Then it must have the form

$$
\{x, y\}=z+z^{2} h, \quad\{y, z\}=z^{2} k, \quad\{x, z\}=x z+z^{2} l,
$$

for some smooth functions $h, k, l$ defined on $U$. Computing the Jacobiator of $x, y$, and $z$, we obtain

$$
J=\{x,\{y, z\}\}+\{z,\{x, y\}\}+\{y,\{z, x\}\}=z^{2}((2-x) k(x, y, 0)+1)+z^{3} a(x, y, z),
$$

where $a$ is a smooth function. In particular, we see that $J$ cannot vanish, since

$$
\frac{\partial^{2} J}{\partial z^{2}}(2, y, 0)=2 \neq 0 .
$$

This example shows that not everything that looks like the first jet of a Poisson structure around $P$ (that is, an extension of the form (6) or (5)) comes from an actual Poisson structure.

On the other hand, if $P$ is a symplectic manifold, the situation changes for the better; every "first jet" of a Poisson structure can be extended to a Poisson structure around $P$. More precisely, consider $\left(S, \omega_{S}\right)$ a symplectic manifold, with $S \subset M$ embedded, and an algebroid structure on $A_{S}:=T_{S}^{*} M$ that fits into the exact sequence

$$
0 \rightarrow T S^{\circ} \rightarrow A_{S} \rightarrow T^{*} S \rightarrow 0 .
$$

Then, using a tubular neighborhood $\mathscr{E}: T_{S} M / T S \rightarrow M$, one can construct a Poisson structure $\pi_{S}^{1}=\pi_{S}^{1}\left(A_{S}, \omega_{S}, \mathscr{E}\right)$ on some open neighborhood of $S$, from which we recover the first-order data: it has $\left(S, \omega_{S}\right)$ as a symplectic leaf, and the algebroid 
structure induced on $T_{S}^{*} M$ is $A_{S}$. This Poisson structure was first constructed by Vorobjev [2001]; we also recommend [Crainic and Mărcuţ 2010] for some different approaches. Applied to different tubular neighborhoods, this construction produces Poisson structures which, when restricted to small enough neighborhoods of $S$, are Poisson diffeomorphic [Vorobjev 2001]. So the isomorphism class of the germ around $S$ of $\pi_{S}^{1}$ doesn't depend on $\mathscr{E}$.

We can view the whole story from a different perspective; start with a Poisson structure $\pi$ on $M$, for which $\left(S, \omega_{S}\right)$ is an embedded symplectic leaf, and denote as usual by $A_{S}$ the Lie algebroid on $T_{S}^{*} M$. For $\mathscr{E}$ a tubular neighborhood of $S$, we call $\pi_{S}^{1}=\pi_{S}^{1}\left(A_{S}, \omega_{S}, \mathscr{E}\right)$ the first-order approximation of $\pi$ around $S$. The first-order approximation is defined on some open neighborhood of $S$ in $M$, and it plays the role of a local normal form for $\pi$ around $S$.

\section{The formal equivalence theorem}

The algebra of formal vector fields. Take the graded Lie algebra $\left(\mathfrak{X}^{\bullet}(M),[\cdot, \cdot]\right)$ of multivector fields on $M$, with the Nijenhuis-Schouten bracket and $\operatorname{deg}(W)=$ $k-1$ for $W \in \mathfrak{X}^{k}(M)$. For a closed, embedded submanifold $P \subset M$, denote by $\mathfrak{X}_{P}^{\bullet}(M)$ the following subalgebra of multivector fields tangent to $P$ :

$$
\mathfrak{X}_{P}^{\bullet}(M):=\left\{u \in \mathfrak{X}^{\bullet}(M) \mid u_{\mid P} \in \mathfrak{X}^{\bullet}(P)\right\} .
$$

The vanishing ideal $I(P) \subset C^{\infty}(M)$ of $P$ induces a filtration $\mathscr{F}_{F}$ on $\mathfrak{X}_{P}^{\bullet}(M)$ :

$$
\begin{gathered}
\mathfrak{X}_{P}^{\bullet}(M) \supset \mathscr{F}_{0}^{\bullet} \supset \mathscr{F}_{1}^{\bullet} \supset \ldots \mathscr{F}_{k}^{\bullet} \supset \mathscr{F}_{k+1}^{\bullet} \supset \ldots, \\
\mathscr{F}_{k}^{\bullet}=I^{k+1}(P) \mathfrak{X}^{\bullet}(M), \quad k \geq 0 .
\end{gathered}
$$

It is readily checked that

$$
\left[\mathscr{F}_{k}, \mathscr{F}_{l}\right] \subset \mathscr{F}_{k+l}, \quad\left[\mathfrak{X}_{P}^{\bullet}(M), \mathscr{F}_{k}\right] \subset \mathscr{F}_{k} .
$$

Let $\hat{\mathfrak{X}}_{P}^{\bullet}(M)$ be the completion of $\mathfrak{X}_{P}^{\bullet}(M)$ with respect to the filtration $\mathscr{F}$, defined by the projective limit

$$
\hat{\mathfrak{X}}_{P}^{\bullet}(M):=\lim _{\leftarrow} \mathfrak{X}_{P}^{\bullet}(M) / \mathscr{F}_{k}^{\bullet} .
$$

By (7), it follows that $\hat{\mathfrak{X}}_{P}^{\bullet}(M)$ inherits a graded Lie algebra structure, such that, for $k \geq 0$, the natural maps

$$
j_{\mid P}^{k}: \hat{\mathfrak{X}}_{P}^{\bullet}(M) \rightarrow \mathfrak{X}_{P}^{\bullet}(M) / \mathscr{F}_{k}^{\bullet}
$$

are Lie algebra homomorphisms. The algebra $\left(\hat{\mathfrak{X}}_{P}^{\bullet}(M),[\cdot, \cdot]\right)$ is called the algebra of formal multivector fields along $P$. Consider also the homomorphism

$$
j_{\mid P}^{\infty}: \mathfrak{X}_{P}^{\bullet}(M) \rightarrow \hat{\mathfrak{X}}_{P}^{\bullet}(M) .
$$


From a version of Borel's Theorem (see, for example, [Moerdijk and Reyes 1991]) about the existence of a smooth section with a specified infinite jet along a submanifold, it follows that $j_{\mid P}^{\infty}$ is surjective. Observe that $\hat{\mathfrak{X}}_{P}^{\bullet}(M)$ inherits a filtration $\hat{\mathscr{F}}$ from $\mathfrak{X}_{P}^{\bullet}(M)$, given by

$$
\hat{\mathscr{F}}_{k}^{\bullet}=j_{\mid P}^{\infty} \mathscr{F}_{k}^{\bullet},
$$

that satisfies the corresponding equations (7).

The adjoint action of an element $X \in \hat{\mathscr{F}}_{1}$

$$
\operatorname{ad}_{X}: \hat{\mathfrak{X}}_{P}^{\bullet}(M) \rightarrow \hat{\mathfrak{X}}_{P}^{\bullet}(M), \quad \operatorname{ad}_{X}(Y):=[X, Y]
$$

increases the degree of the filtration by 1 . Therefore the partial sums

$$
\sum_{i=0}^{n} \frac{\operatorname{ad}_{X}^{i}}{i !}(Y)
$$

are constant modulo $\hat{\mathscr{F}}_{k}$ for $n \geq k$ and all $Y \in \hat{\mathfrak{X}}_{P}^{\bullet}(M)$. This and the completeness of the filtration on $\hat{\mathscr{F}}$ show that the exponential of $\operatorname{ad}_{X}$

$$
e^{\operatorname{ad}_{X}}: \hat{\mathfrak{X}}_{P}^{\bullet}(M) \rightarrow \hat{\mathfrak{X}}_{P}^{\bullet}(M), \quad e^{\operatorname{ad}_{X}}(Y):=\sum_{n \geq 0} \frac{\operatorname{ad}_{X}^{n}}{n !}(Y)
$$

is well-defined. It is readily checked that $e^{\operatorname{ad}_{X}}$ is a graded Lie algebra isomorphism with inverse $e^{-\operatorname{ad}_{X}}$ and that it preserves the filtration. We need the following geometric interpretation of these isomorphisms.

Lemma 3.1. For every $X \in \hat{\mathscr{F}}_{1}^{1}$, there exists $\psi: M \rightarrow M$ a diffeomorphism of $M$, with $\psi_{\mid P}=\operatorname{id}_{P}$ and $d \psi_{\mid P}=\operatorname{id}_{T_{P} M}$, such that for every $W \in \mathfrak{X}_{P}^{\bullet}(M)$, we have

$$
j_{\mid P}^{\infty}\left(\psi^{*}(W)\right)=e^{\operatorname{ad}_{X}}\left(j_{\mid P}^{\infty}(W)\right) .
$$

Proof. By Borel's Theorem, there is a vector field $V$ on $M$ such that $X=j_{\mid P}^{\infty}(V)$. We claim that $V$ can be chosen to be complete. Let $g$ be a complete metric on $M$ and let $\phi: M \rightarrow[0,1]$ be a smooth function that satisfies $\phi=1$ on the set $\left\{x \mid g_{x}\left(V_{x}, V_{x}\right) \leq \frac{1}{2}\right\}$ and $\phi=0$ on the set $\left\{x \mid g_{x}\left(V_{x}, V_{x}\right) \geq 1\right\}$. Since $V_{\mid P}=0$, it follows that $\phi V$ has the same germ as $V$ around $P$, and therefore $j_{\mid P}^{\infty}(\phi V)=X$. On the other hand, since $\phi V$ is bounded, it is complete, so replace $V$ by $\phi V$.

We show that $\psi:=\mathrm{Fl}_{V}$, the flow of $V$ at time 1 , satisfies all requirements. Since $j_{\mid P}^{1}(V)=0$, it is clear that $\psi_{\mid P}=\mathrm{id}_{P}$ and $d \psi_{\mid P}=\operatorname{id}_{T_{P} M}$.

Consider $W \in \mathfrak{X}_{P}^{\bullet}(M)$, and denote by $W_{s}:=\mathrm{Fl}_{s V}^{*}(W)$ the pullback of $W$ by the flow of $V$ at time $s$. Since $W_{s}$ satisfies the differential equation $d W_{s} / d s=\left[V, W_{s}\right]$, 
a simple computation gives

$$
\frac{d}{d s}\left(\sum_{i=0}^{k} \frac{(-s)^{i} \operatorname{ad}_{V}^{i}}{i !}\left(W_{s}\right)\right)=\frac{(-s)^{k} \operatorname{ad}_{V}^{k+1}}{k !}\left(W_{s}\right) .
$$

This shows that the sum

$$
\sum_{i=0}^{k} \frac{(-s)^{i} \operatorname{ad}_{V}^{i}}{i !}\left(W_{s}\right)
$$

modulo $\mathscr{F}_{k+1}$ is independent of $s$, and therefore

$$
W-\sum_{i=0}^{k} \frac{(-1)^{i} \operatorname{ad}_{V}^{i}}{i !}\left(\psi^{*}(W)\right) \in \mathscr{F}_{k+1} .
$$

Applying $j_{\mid P}^{\infty}$ to this equation yields

$$
j_{\mid P}^{\infty}(W)-\sum_{i=0}^{k} \frac{(-1)^{i} \operatorname{ad}_{X}^{i}}{i !} j_{\mid P}^{\infty}\left(\psi^{*}(W)\right) \in \hat{\mathscr{F}}_{k+1},
$$

and hence we conclude

$$
j_{\mid P}^{\infty}(W)=e^{-\operatorname{ad}_{X}} j_{\mid P}^{\infty}\left(\psi^{*}(W)\right) .
$$

The cohomology of the restricted algebroid. Let $(M, \pi)$ be a Poisson manifold and $P \subset M$ a closed, embedded Poisson submanifold. The cohomologies we are considering are all versions of the Poisson cohomology $H_{\pi}^{\bullet}(M)$, computed by the complex $\mathfrak{X}^{\bullet}(M)$ of multivector fields on $M$ and differential $d_{\pi}=[\pi, \cdot]$. Since $P$ is a Poisson submanifold, we have that $\left[\pi, I(P) \mathfrak{X}^{\bullet}(M)\right] \subset I(P) \mathfrak{X}^{\bullet}(M)$, and more generally, it follows that $I^{k}(P) \mathfrak{X}^{\bullet}(M)$ forms a subcomplex. Taking consecutive quotients, we obtain the complexes

$$
\left(I^{k}(P) \mathfrak{X}^{\bullet}(M) / I^{k+1}(P) \mathfrak{X}^{\bullet}(M), d_{\pi}^{k}\right),
$$

with differential $d_{\pi}^{k}$ induced by $[\pi, \cdot]$. For $k=0$, we obtain the Poisson cohomology relative to $P$. Observe that the differential on these complexes depends only on the first jet of $\pi$ along $P$, and therefore, following the philosophy of Section 2, it can be described only in terms of the algebroid $A_{P}$.

Proposition 3.2. The following two complexes are isomorphic:

$$
\left(I^{k}(P) \mathfrak{X}^{\bullet}(M) / I^{k+1}(P) \mathfrak{X}^{\bullet}(M), d_{\pi}^{k}\right) \cong\left(\Omega^{\bullet}\left(A_{P}, \varphi^{k}\left(T P^{\circ}\right)\right), d_{\nabla^{k}}\right) \quad \text { for all } k \geq 0 .
$$

Proof. Since the space of sections of $T P^{\circ}$ is spanned by differentials of elements in $I(P)$, it is easy to see that the map given by

$$
\tau_{k}: I^{k}(P) \mathfrak{X}^{\bullet}(M) \rightarrow \Omega^{\bullet}\left(A_{P}, \mathscr{Y}^{k}\left(T P^{\circ}\right)\right)=\Gamma\left(\Lambda^{\bullet}\left(T_{P} M\right) \otimes \mathscr{S}^{k}\left(T P^{\circ}\right)\right),
$$




$$
\tau_{k}\left(f_{1} \ldots f_{k} W\right)=W_{\mid P} \otimes d f_{1 \mid P} \odot \cdots \odot d f_{k \mid P},
$$

where $f_{1}, \ldots, f_{k} \in I(P)$ and $W \in \mathfrak{X}^{\bullet}(M)$, is well-defined and surjective. Also, its kernel is precisely $I^{k+1}(P) \mathfrak{X}^{\bullet}(M)$. Hence, it remains to prove that

$$
\tau_{k}([\pi, W])=d_{\nabla^{k}}\left(\tau_{k}(W)\right) \text { for all } W \in I^{k}(P) \mathfrak{X}^{\bullet}(M) .
$$

Recall that the algebroid $A_{P}$ has anchor $\rho=\pi_{\mid P}^{\sharp}$ and bracket determined by

$$
\left[d \phi_{\mid P}, d \psi_{\mid P}\right]_{P}:=d\{\phi, \psi\}_{\mid P} \quad \text { for all } \phi, \psi \in C^{\infty}(M) .
$$

Also, for $k=0$, we have that $\nabla^{0}$ is given by

$$
\nabla^{0}: \Gamma\left(A_{P}\right) \times C^{\infty}(P) \rightarrow C^{\infty}(P), \quad \nabla_{\eta}^{0}(h)=L_{\rho(\eta)}(h) .
$$

Since both differentials $d_{\pi}$ and $d_{\nabla^{k}}$ act by derivations and $\nabla^{k}$ is obtained by extending $\nabla^{1}$ by derivations, it suffices to prove (8) for $\phi \in C^{\infty}(M)$ and $X \in \mathfrak{X}^{1}(M)$ (with $k=0$ ), and for $f \in I(P)$ (with $k=1$ ).

Let $\phi \in C^{\infty}(M)$ and $\eta \in \Gamma\left(A_{P}\right)$. Since $\pi$ is tangent to $P$, we obtain

$$
\tau_{0}([\pi, \phi])(\eta)=[\pi, \phi]_{\mid P}(\eta)=d \phi_{\mid P}\left(\pi_{\mid P}^{\sharp}(\eta)\right)=L_{\rho(\eta)}\left(\tau_{0}(\phi)\right)=d_{\nabla^{0}}\left(\tau_{0}(\phi)\right)(\eta) .
$$

Let $X \in \mathfrak{X}^{1}(M)$ and $\phi, \psi \in C^{\infty}(M)$, and define $\eta:=d \phi_{\mid P}$ for $\theta:=d \psi_{\mid P} \in \Gamma\left(A_{P}\right)$. Then

$$
\begin{aligned}
\tau_{0}([\pi, X])(\eta, \theta)= & {[\pi, X]_{\mid P}\left(d \phi_{\mid P}, d \psi_{\mid P}\right) } \\
= & (\{X(\phi), \psi\}+\{\phi, X(\psi)\}-X(\{\phi, \psi\}))_{\mid P} \\
= & \pi_{\mid P}^{\sharp}\left(d \phi_{\mid P}\right)\left(X_{\mid P}\left(d \psi_{\mid P}\right)\right) \\
& \quad-\pi_{\mid P}^{\sharp}\left(d \psi_{\mid P}\right)\left(X_{\mid P}\left(d \phi_{\mid P}\right)\right)-X_{\mid P}\left(d\{\phi, \psi\}_{\mid P}\right) \\
= & L_{\rho(\eta)}\left(\tau_{0}(X)(\theta)\right)-L_{\rho(\theta)}\left(\tau_{0}(X)(\eta)\right)-\tau_{0}(X)\left([\eta, \theta]_{P}\right) \\
= & d_{\nabla^{0}}\left(\tau_{0}(X)\right)(\eta, \theta),
\end{aligned}
$$

and thus (8) holds for $X$.

Consider now $f \in I(P)$ and $\eta:=d \phi_{\mid P} \in \Gamma\left(A_{P}\right)$, with $\phi \in C^{\infty}(M)$. The formula defining $\tau_{k}$ implies that for every $W \in I^{k}(P) \mathfrak{X}^{\bullet}(M)$, we have

$$
\tau_{k}\left(i_{d \phi}(W)\right)=i_{d \phi \mid P} \tau_{k}(W) .
$$

Using this, the following computation finishes the proof:

$$
\begin{aligned}
\tau_{1}([\pi, f])(\eta) & =\tau_{1}([\pi, f](d \phi))=\tau_{1}(\{\phi, f\})=d\{\phi, f\}_{\mid P} \\
& =\left[\eta, d f_{\mid P}\right]_{P}=\nabla_{\eta}^{1}(\tau(f))=d_{\nabla^{1}}(\tau(f))(\eta) .
\end{aligned}
$$


Proof of Theorem 1.1. By replacing $M$ with a tubular neighborhood of $P$, we can assume that $P$ is closed in $M$. Write

$$
\gamma:=j_{\mid P}^{\infty} \pi_{1}, \quad \gamma^{\prime}:=j_{\mid P}^{\infty} \pi_{2} \in \hat{\mathfrak{X}}_{P}^{2}(M) .
$$

By Proposition 3.2, we can recast the hypothesis as

$$
[\gamma, \gamma]=0, \quad\left[\gamma^{\prime}, \gamma^{\prime}\right]=0, \quad \gamma-\gamma^{\prime} \in \hat{\mathscr{F}}_{1}, \quad H^{2}\left(\hat{\mathscr{F}}_{k}^{\bullet} / \hat{\mathscr{F}}_{k+1}^{\bullet}, d_{\gamma}\right)=0
$$

for all $k \geq 1$, where $d_{\gamma}:=\operatorname{ad}_{\gamma}$. All these conditions are expressed in terms of the

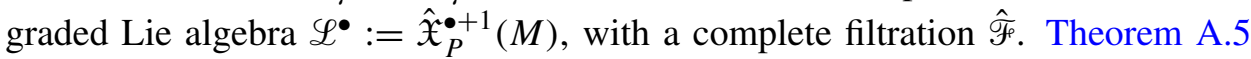
in the Appendix shows that there exists a formal vector field $X \in \hat{\mathscr{F}}_{1}^{1}$ such that $\gamma=e^{\operatorname{ad}_{X}}\left(\gamma^{\prime}\right)$. By Lemma 3.1, there exists a diffeomorphism $\psi$ of $M$, such that $j_{\mid P}^{\infty}\left(\psi^{*}(W)\right)=e^{\operatorname{ad}_{X}} j_{\mid P}^{\infty}(W)$, for all $W \in \mathfrak{X}_{P}^{\bullet}(M)$. This concludes the proof, since

$$
j_{\mid P}^{\infty}\left(\psi^{*}\left(\pi_{2}\right)\right)=e^{\operatorname{ad}_{X}} j_{\mid P}^{\infty}\left(\pi_{2}\right)=e^{\operatorname{ad}_{X}}\left(\gamma^{\prime}\right)=\gamma=j_{\mid P}^{\infty}\left(\pi_{1}\right) .
$$

Existence of Poisson structures with a specified infinite jet. This proof can be applied to obtain a result on existence of Poisson bivectors with a specified infinite jet. Let $S$ be a closed embedded submanifold of $M$. An element $\hat{\pi} \in \hat{\mathfrak{X}}_{S}^{2}(M)$, satisfying $[\hat{\pi}, \hat{\pi}]=0$, is called a formal Poisson bivector. Observe that

$$
\hat{\pi}_{\mid S}:=\hat{\pi} \quad \bmod \hat{\mathscr{F}}_{0} \in \mathfrak{X}^{2}(S)
$$

is a Poisson structure on $S$. We call $S$ a symplectic leaf on $\hat{\pi}$ if $\hat{\pi}_{\mid S}$ is nondegenerate. Assuming that $S$ is a symplectic leaf of $\hat{\pi}$, by the discussion in Section 2, the first jet of $\hat{\pi}$,

$$
j_{\mid S}^{1}(\hat{\pi})=\hat{\pi} \quad \bmod \hat{\mathscr{F}}_{1},
$$

determines a Lie algebroid $A_{S}$ on $T_{S}^{*} M$, and thus can be used to construct a Poisson bivector $\pi_{S}^{1}$ on some open neighborhood $U$ of $S$, whose first jet coincides with that of $\hat{\pi}$. If the cohomology groups

$$
H^{2}\left(A_{S} ; \mathscr{Y}^{k}\left(T S^{\circ}\right)\right)
$$

vanish for all $k \geq 2$, then by the proof of Theorem 1.1, there exists a formal vector field $X \in \hat{\mathscr{F}}_{1}^{1}$ such that $e^{\operatorname{ad}_{X}}\left(j_{\mid S}^{\infty} \pi_{S}^{1}\right)=\hat{\pi}$. By Lemma 3.1, we find a diffeomorphism $\psi: \mathcal{U} \rightarrow \mathcal{U}$ such that

$$
j_{\mid S}^{\infty}\left(\psi^{*}\left(\pi_{S}^{1}\right)\right)=e^{\operatorname{ad}_{X}}\left(j_{\mid S}^{\infty} \pi_{S}^{1}\right)=\hat{\pi} .
$$

Thus $\pi:=\psi^{*}\left(\pi_{S}^{1}\right)$ gives a Poisson structure defined on an open neighborhood of $S$ whose infinite jet is $\hat{\pi}$. Hence we have proved the following statement. 
Corollary 3.3. Let $\hat{\pi} \in \hat{\mathfrak{X}}_{S}^{2}(M)$ be a formal Poisson structure for which $S$ is a symplectic leaf. If for any $k \geq 2$, the algebroid $A_{S}$ induced by $j_{\mid S}^{1} \hat{\pi}$ satisfies

$$
H^{2}\left(A_{S} ; \mathscr{Y}^{k}\left(T S^{\circ}\right)\right)=0,
$$

then there exists a Poisson structure $\pi$ defined on some open neighborhood of $S$ such that $\hat{\pi}=j_{\mid S}^{\infty} \pi$.

\section{Proofs of the criteria}

Here we explain and prove the corollaries from the Introduction.

Integration of Lie algebroids and differentiable cohomology. We recall some properties of Lie groupoids and Lie algebroids; see [Mackenzie 1987; Moerdijk and Mrčun 2003] for the general theory. A Lie groupoid over a manifold $B$ is denoted by $\mathscr{G}$, the source and target maps by $s, t: \mathscr{G} \rightarrow B$, and the unit map by $u: B \rightarrow \mathscr{G}$. To a Lie groupoid $\mathscr{G}$ one can associate a Lie algebroid $A(\mathscr{G}) \rightarrow B$, which is the infinitesimal counterpart of $\mathscr{G}$. A Lie algebroid $\mathscr{A}$ is called integrable if $\mathscr{A} \cong A(\mathscr{G})$ for some Lie groupoid $\mathscr{G}$. The relation between Lie algebroids and Lie groupoids is similar to that between Lie algebras and Lie groups, the most significant difference being that not every Lie algebroid is integrable.

Recall that a transitive Lie algebroid is a Lie algebroid $A \rightarrow B$ with surjective anchor. For example, if $S \subset M$ is a symplectic leaf of a Poisson manifold $(M, \pi)$, then the Lie algebroid $A_{S}$ is transitive. A Lie groupoid $\mathscr{G}$ is called transitive if the map $(s, t): \mathscr{G} \rightarrow M \times M$ is a surjective submersion. The Lie algebroid of a transitive Lie groupoid is transitive. Conversely, if the base $B$ of a transitive Lie algebroid $\mathscr{A}$ is connected, and $\mathscr{A}$ is integrable, then any Lie groupoid $\mathscr{G}$ integrating it is transitive. Every transitive Lie groupoid is a gauge groupoid; that is, it is of the form $P \times{ }_{G} P$, where $G$ is a Lie group and $p: P \rightarrow B$ is a principal $G$-bundle. For $P$ one can take any $s$-fiber $s^{-1}(x)$ of $\varphi$ for $x \in B$, and $G:=s^{-1}(x) \cap t^{-1}(x)$. We can recover $\mathscr{A}$ from $P$ as follows: as a bundle $\mathscr{A}=T P / G$, the Lie bracket is induced by the identification

$$
\Gamma(\mathscr{A})=\mathfrak{X}(P)^{G},
$$

and the anchor is given by $d p$. We will also say, about a principle $G$-bundle $P$ for which $A \approx T P / G$, that it integrates $A$. As for Lie algebras, if a transitive Lie algebroid with connected base is integrable, then, up to isomorphism, there exists a unique 1-connected principal bundle integrating it [Mackenzie 1987].

Let $S \subset M$ be a symplectic leaf of a Poisson manifold $(M, \pi)$, and assume that the transitive algebroid $A_{S}$ is integrable. The connected and simply connected principal bundle $P \rightarrow S$ for which $P \times{ }_{G} P$ integrates $A_{S}$ is called the Poisson homotopy cover of $S$. We say that $P$ is smooth if $A_{S}$ is integrable; this terminology 
is justified by the fact that $P$ exists also in the nonintegrable case as a topological principal bundle over $S$ [Crainic and Fernandes 2003].

Let $\mathscr{A}$ be a transitive Lie algebroid with connected base space $B$, and denote by $\mathfrak{g} \subset \mathscr{A}$ the kernel of the anchor. On each fiber of $\mathfrak{g}$, the Lie bracket restricts to a Lie algebra structure $\left(\mathfrak{g}_{x},[\cdot, \cdot]_{x}\right)$, and this Lie algebra is called the isotropy Lie algebra at $x$. In the integrable case, when $\mathscr{A}=A(\mathscr{G})$, the isotropy Lie algebra coincides with the Lie algebra of the isotropy group $G_{x}:=s^{-1}(x) \cap t^{-1}(x)$. In the case of a symplectic leaf $S \subset M$ of a Poisson manifold, the kernel of the anchor of the Lie algebroid $A_{S}$ is given by $\mathfrak{g}:=T S^{\circ}$.

A Lie groupoid $\mathscr{G}$ is called proper if $(s, t): \mathscr{G} \rightarrow B \times B$ is a proper map.

A representation of a Lie groupoid $\mathscr{G}$ over $B$ is a vector bundle $E \rightarrow B$ and a smooth linear action $g: E_{x} \rightarrow E_{y}$ for every arrow $g: x \rightarrow y$ satisfying the obvious identities. A representation $E$ of $\varphi$ can be differentiated to a representation of its Lie algebroid $A(\mathscr{G})$ on the same vector bundle $E$. If the $s$-fibers of $\mathscr{G}$ are connected and simply connected, then every representation of $A(G)$ comes from a representation of $\mathscr{G}$ [Crainic and Fernandes 2003, Proposition 2.2], and in our applications this is usually the case.

The differentiable cohomology of a Lie groupoid $\varphi$ with coefficients in a representation $E \rightarrow B$ is computed by the complex $\mathscr{C}_{\mathrm{diff}}^{p}(\mathscr{G} ; E)$ of smooth maps $c: \varphi^{(p)} \rightarrow E$, where

$$
\mathscr{G}^{(p)}:=\left\{\left(g_{1}, \ldots, g_{p}\right) \in \mathscr{G}^{p} \mid s\left(g_{i}\right)=t\left(g_{i+1}\right), i=1, \ldots, p-1\right\}
$$

with $c\left(g_{1}, \ldots, g_{p}\right) \in E_{t\left(g_{1}\right)}$, and with differential given by

$$
\begin{aligned}
d c\left(g_{1}, \ldots, g_{p+1}\right) & =g_{1} c\left(g_{2}, \ldots, g_{p+1}\right) \\
+ & \sum_{i=1}^{p}(-1)^{i} c\left(g_{1}, \ldots, g_{i} g_{i+1}, \ldots, g_{p+1}\right)+(-1)^{p+1} c\left(g_{1}, \ldots, g_{p}\right) .
\end{aligned}
$$

The resulting cohomology groups are denoted $H_{\text {diff }}^{\bullet}(\mathscr{G}, E)$. For more details on this subject, see [Haefliger 1979].

In the following proposition we list some results from [Crainic 2003] that are needed in the proofs of the corollaries from the Introduction.

Proposition 4.1. Let $G$ be a Lie groupoid over B with Lie algebroid $A$, and let $E \rightarrow B$ be a representation of $\varphi$.

(1) If the s-fibers of 9 are cohomologically 2-connected, then

$$
H^{2}(\mathscr{A} ; E) \cong H_{\text {diff }}^{2}(\varphi ; E) .
$$

(2) If $\varphi$ is proper, then $H_{\text {diff }}^{2}(\varphi ; E)=0$.

(3) If $\varphi_{\text {is }}$ transitive, then $H_{\mathrm{diff}}^{2}\left(\varphi_{;} ; E\right) \cong H_{\mathrm{diff}}^{2}\left(\mathscr{G}_{x} ; E_{x}\right)$, where $x \in B$ and $\mathscr{G}_{x}:=$ $s^{-1}(x) \cap t^{-1}(x)$. 
Proof. (1) is a particular case of [Crainic 2003, Theorem 4], and (2) follows from [Crainic 2003, Proposition 1]. Since $\mathscr{G}$ is transitive, it is Morita equivalent to $\mathscr{G}_{x}$ [Moerdijk and Mrčun 2003]; by [Crainic 2003, Theorem 1], a Morita equivalence induces an isomorphism in cohomology, and this proves (3).

Proof of Corollary 1.2. Recall that the cotangent Lie algebroid of $\left(\mathfrak{g}^{*}, \pi_{\text {lin }}\right)$ is isomorphic to the action Lie algebroid $\mathfrak{g} \ltimes \mathfrak{g}^{*} \rightarrow \mathfrak{g}^{*}$ for the coadjoint action of $\mathfrak{g}$ on $\mathfrak{g}^{*}$, and that it is integrable by the action groupoid $G \ltimes \mathfrak{g}^{*}$, where $G$ denotes the compact, connected and simply connected Lie group of $\mathfrak{g}$. Also, the symplectic leaves of $\left(\mathfrak{g}^{*}, \pi_{\text {lin }}\right)$ are the orbits of the action of $G$. So, because $\mathbb{S}(\mathfrak{g})$ is $G$ invariant, it is a union of symplectic leaves, and therefore a Poisson submanifold. The algebroid $A_{\mathbb{S}(\mathfrak{g})}$ is isomorphic to the action algebroid $\mathfrak{g} \ltimes \mathbb{S}(\mathfrak{g})$, and therefore it is integrable by the action groupoid $G \ltimes \mathfrak{S}(\mathfrak{g})$. Since $G$ is simply connected, it follows that $H_{d R}^{2}(G)=0$ [Duistermaat and Kolk 2000, Theorem 1.14.2]. On the other hand, all $s$-fibers of $G \ltimes S(\mathfrak{S})$ are diffeomorphic to $G$, and so the assumptions of Proposition 4.1(1) are satisfied, and therefore, for any representation $E \rightarrow \mathbb{S}(\mathfrak{g})$ of $G \ltimes \mathbb{S}(\mathfrak{g})$, we have

$$
H^{2}(\mathfrak{g} \ltimes \mathbb{S}(\mathfrak{g}) ; E) \cong H_{\text {diff }}^{2}(G \ltimes \mathbb{S}(\mathfrak{g}) ; E) .
$$

Since $G \ltimes \mathbb{S}(\mathfrak{g})$ is compact, it is proper, and hence by Proposition 4.1(2), we have $H_{\text {diff }}^{2}(G \ltimes S(\mathfrak{g}) ; E)=0$ for every representation $E$. Now the corollary follows from Theorem 1.1.

Proof of Corollary 1.4. Denote by $P$ the Poisson homotopy cover of $S$ with structure group $G$. By hypothesis, $P$ is smooth, simply connected and with vanishing second de Rham cohomology group. Let $\mathscr{G}:=P \times_{G} P$ be the gauge groupoid of $P$. Since every $s$-fiber of $\mathscr{G}$ is diffeomorphic to $P, \mathscr{G}$ satisfies the assumptions of Proposition 4.1(1), and therefore

$$
H^{2}\left(A_{S} ; \varphi^{k}\left(T S^{\circ}\right)\right) \cong H_{\mathrm{diff}}^{2}\left(\varphi ; \varphi^{k}\left(T S^{\circ}\right)\right) .
$$

Since $\mathscr{G}$ is transitive, by Proposition 4.1(3), we have

$$
H_{\text {diff }}^{2}\left(\varphi ; \varphi^{k}\left(T S^{\circ}\right)\right) \cong H_{\text {diff }}^{2}\left(G ; \varphi^{k}\left(T_{x} S^{\circ}\right)\right) .
$$

Since $T_{x} S^{\circ} \cong \mathfrak{g}$ as $G$ representations (both integrate the adjoint representation of $\mathfrak{g}$ ), the proof follows from Theorem 1.3.

Proof of Corollary 1.5. This follows from Corollary 1.4, because the differentiable cohomology of compact Lie groups vanishes, by Proposition 4.1(2).

Proof of Corollary 1.6. Let $x \in S$, and denote by $\mathfrak{g}_{x}:=T_{x} S^{\circ}$ the isotropy Lie algebra of the transitive algebroid $A_{S}$. By hypothesis, $\mathfrak{g}_{x}$ is reductive; that is, it splits as a direct product of a semisimple Lie algebra and its center $\mathfrak{g}_{x}=\mathfrak{s}_{x} \oplus \mathfrak{z}_{x}$, 
where $\mathfrak{s}_{x}=\left[\mathfrak{g}_{x}, \mathfrak{g}_{x}\right]$ and $\mathfrak{z}_{x}=Z\left(\mathfrak{g}_{x}\right)$ is the center of $\mathfrak{g}_{x}$. Since $\mathfrak{g}=T S^{\circ}$ is a Lie algebra bundle, it follows that this splitting is in fact global:

$$
\mathfrak{g}=[\mathfrak{g}, \mathfrak{g}] \oplus Z(\mathfrak{g})=\mathfrak{s} \oplus \mathfrak{z} .
$$

Since $\mathfrak{s}=[\mathfrak{g}, \mathfrak{g}]$ is an ideal of $A_{S}$, we obtain a short exact sequence of algebroids

$$
0 \rightarrow \mathfrak{s} \rightarrow A_{S} \rightarrow A_{S}^{\mathrm{ab}} \rightarrow 0,
$$

with $A_{S}^{\mathrm{ab}}=A_{S} /[\mathfrak{g}, \mathfrak{g}]$. Similar to the spectral sequence for Lie algebra extensions [Hochschild and Serre 1953], there is a spectral sequence for extensions of Lie algebroids [Mackenzie 1987, Theorem 5.5 and the remark following it], which in our case converges to $H^{\bullet}\left(A_{S} ; \mathscr{Y}^{k}(\mathfrak{g})\right)$, with

$$
E_{2}^{p, q}=H^{p}\left(A_{S}^{\mathrm{ab}} ; H^{q}\left(\mathfrak{s} ; \mathscr{Y}^{k}(\mathfrak{g})\right)\right) \Rightarrow H^{p+q}\left(A_{S} ; \mathscr{Y}^{k}(\mathfrak{g})\right) .
$$

Since $\mathfrak{s}$ is in the kernel of the anchor, $H^{q}\left(\mathfrak{s} ; \mathscr{Y}^{k}(\mathfrak{g})\right)$ is indeed a vector bundle, with fiber $H^{q}\left(\mathfrak{s} ; \mathscr{Y}^{k}(\mathfrak{g})\right)_{x}=H^{q}\left(\mathfrak{s}_{x} ; \mathscr{Y}^{k}\left(\mathfrak{g}_{x}\right)\right)$, and it inherits a representation of $A_{S}^{\mathrm{ab}}$. Since $\mathfrak{s}_{x}$ is semisimple, by the Whitehead Lemma we have that $H^{1}\left(\mathfrak{s}_{x} ; \mathscr{S}^{k}\left(\mathfrak{g}_{x}\right)\right)=0$ and $H^{2}\left(\mathfrak{s}_{x} ; \varphi^{k}\left(\mathfrak{g}_{x}\right)\right)=0$. Therefore,

$$
H^{2}\left(A_{S} ; \mathscr{Y}^{k}(\mathfrak{g})\right) \cong H^{2}\left(A_{S}^{\mathrm{ab}} ; \mathscr{Y}^{k}(\mathfrak{g})^{\mathfrak{s}}\right),
$$

where $\mathscr{S}^{k}\left(\mathfrak{g}_{x}\right)^{\mathfrak{s}_{x}}$ is the $\mathfrak{s}_{x}$-invariant part of $\mathscr{S}^{k}\left(\mathfrak{g}_{x}\right)$. By hypothesis, $A_{S}^{\text {ab }}$ is integrable by a principle bundle $P^{\mathrm{ab}}$ that is simply connected and that has vanishing second de Rham cohomology and compact structure group $T$. Therefore, by (9) and by applying Proposition 4.1(1), (2) and (3), we obtain that

$$
\begin{aligned}
H^{2}\left(A_{S} ; \mathscr{\varphi}^{k}(\mathfrak{g})\right) & \cong H^{2}\left(A_{S}^{\mathrm{ab}} ; \mathscr{\varphi}^{k}(\mathfrak{g})^{\mathfrak{s}}\right) \cong H_{\mathrm{diff}}^{2}\left(P^{\mathrm{ab}} \times_{T} P^{\mathrm{ab}} ; \mathscr{\varphi}^{k}(\mathfrak{g})^{\mathfrak{s}}\right) \\
& \cong H_{\text {diff }}^{2}\left(T ; \mathscr{Y}^{k}\left(\mathfrak{g}_{x}\right)^{\mathfrak{s}_{x}}\right)=0 .
\end{aligned}
$$

Theorem 1.3 finishes the proof.

Proof of Corollary 1.7. Assume that $\mathfrak{g}_{x}$ is semisimple, $\pi_{1}(S, x)$ is finite, and $\pi_{2}(S, x)$ is a torsion group. With the notation above, we have $A_{S}^{\mathrm{ab}} \cong T S$. Also, $T S$ is integrable, and the simply connected principal bundle integrating it is $\widetilde{S}$, the universal cover of $S$. Finiteness of $\pi_{1}(S)$ is equivalent to compactness of the structure group of $\widetilde{S}$. By the Hurewicz theorem, we have $H_{2}(\widetilde{S}, \mathbb{Z}) \cong \pi_{2}(\widetilde{S})$, and since $\pi_{2}(\widetilde{S})=\pi_{2}(S)$ is torsion, we have $H_{\mathrm{dR}}^{2}(\widetilde{S})=0$. So the result follows from Corollary 1.6.

\section{Appendix: Equivalence of MC-elements in complete GLAs}

Here we discuss some general facts about graded Lie algebras endowed with a complete filtration, with the aim of proving a criterion for equivalence of MaurerCartan elements (Theorem A.5), which is used in the proof of Theorem 1.1. Some 
of the constructions given here can be also found in [Bursztyn et al. 2009, Appendix B.1] in the more general setting of differential graded Lie algebras with a complete filtration. In fact, all our constructions can be adapted to this setup, including in particular Theorem A.5. The analog of Theorem A.5 in the case of differential graded associative algebras is in [Abad et al. 2010, Appendix A].

Definitions A.1. (1) A graded Lie algebra $\left(\mathscr{L}^{\bullet},[\cdot, \cdot]\right)$ (or GLA) consists of a $\mathbb{Z}$-graded vector space $\mathscr{L}^{\bullet}$ endowed with a graded bracket $[\cdot, \cdot]: \mathscr{L}^{p} \times \mathscr{L}^{q} \rightarrow$ $\mathscr{L}^{p+q}$, which is graded commutative and satisfies the graded Jacobi identity:

$[X, Y]=-(-1)^{|X||Y|}[Y, X], \quad[X,[Y, Z]]=[[X, Y], Z]+(-1)^{|X||Y|}[Y,[X, Z]]$.

(2) An element $\gamma \in \mathscr{L}^{1}$ satisfying $[\gamma, \gamma]=0$ is called a Maurer-Cartan element.

(3) A filtration on a GLA is a decreasing sequence of homogeneous subspaces

$$
\mathscr{L}^{\bullet} \supset \mathscr{F}_{0} \mathscr{L}^{\bullet} \supset \cdots \supset \mathscr{F}_{n} \mathscr{L}^{\bullet} \supset \mathscr{F}_{n+1} \mathscr{L}^{\bullet} \supset \cdots
$$

satisfying

$$
\left[\mathscr{F}_{n} \mathscr{L}, \mathscr{F}_{m} \mathscr{L}\right] \subset \mathscr{F}_{n+m} \mathscr{L}, \quad\left[\mathscr{L}, \mathscr{F}_{n} \mathscr{L}\right] \subset \mathscr{F}_{n} \mathscr{L} .
$$

(4) A filtration $\mathscr{F} \mathscr{L}$ is called complete if $\mathscr{L}$ is isomorphic to the projective limit $\underset{\lim }{\leftarrow} \mathscr{L} / \mathscr{F}_{n} \mathscr{L}$.

An example of a GLA with a complete filtration appeared in Section 3: starting from a manifold $M$ and a closed embedded submanifold $P \subset M$, we constructed $\left(\hat{\mathfrak{X}}_{P}^{\bullet+1}(M),[\cdot, \cdot]\right)$, the algebra of formal vector fields along $P$, with filtration given by the powers of the vanishing ideal of $P$. So, the index of the filtration plays the role of the order to which elements vanish along $P$.

For a general GLA with a complete filtration $\mathscr{F} \mathscr{L}$, define the order of an element as follows:

$$
\begin{aligned}
& \mathcal{O}: \mathscr{L} \rightarrow\{0,1, \ldots, \infty\}, \\
& \mathcal{O}(X)= \begin{cases}0 & \text { if } X \in \mathscr{L} \backslash \mathscr{F}_{1} \mathscr{L}, \\
n & \text { if } X \in \mathscr{F}_{n} \mathscr{L} \backslash \mathscr{F}_{n+1} \mathscr{L}, \\
\infty & \text { if } X=0 .\end{cases}
\end{aligned}
$$

The order has the following properties, which follow from those of the filtration:

- $O(X)=\infty$ if and only if $X=0$,

- $\mathcal{O}(X+Y) \geq \mathcal{O}(X) \wedge \mathcal{O}(Y)^{2}$,

- $\mathcal{O}(\alpha X) \geq \mathcal{O}(X)$ for all $\alpha \in \mathbb{R}$,

- $\mathscr{O}([X, Y]) \geq \mathscr{O}(X)+\mathscr{O}(Y)$.

${ }^{2} u \wedge v$ denotes $\min \{u, v\}$. 
The completeness assumption on the filtration implies the following property:

Lemma A.2. Let $\left\{X_{n}\right\}_{n \geq 0} \in \mathscr{L}$ be a sequence of elements such that

$$
\lim _{n \rightarrow \infty} \mathcal{O}\left(X_{n}\right)=\infty
$$

Then there exists a unique element $X \in \mathscr{L}$, denoted $X:=\sum_{n \geq 0} X_{n}$, such that

$$
X-\sum_{k=0}^{n} X_{k} \in \mathscr{F}_{m} \mathscr{L}
$$

for all $n$ big enough.

Note that $\mathfrak{g}(\mathscr{L}):=\mathscr{F}_{1} \mathscr{L}^{0}$ forms a Lie subalgebra of $\mathscr{L}^{0}$. Elements $X \in \mathfrak{g}(\mathscr{L})$ satisfy $\mathscr{O}\left(\operatorname{ad}_{X}(Y)\right) \geq \mathcal{O}(Y)+1$ for all $Y \in \mathscr{L}$, and therefore, by Lemma A.2, the exponential of $\operatorname{ad}_{X}$ is well defined, and it is a GLA-automorphism of $\mathscr{L}^{\bullet}$, written

$$
\operatorname{Ad}\left(e^{X}\right): \mathscr{L}^{\bullet} \rightarrow \mathscr{L}^{\bullet}, \quad \operatorname{Ad}\left(e^{X}\right) Y:=e^{\operatorname{ad}_{X}}(Y)=\sum_{n \geq 0} \frac{\operatorname{ad}_{X}^{n}}{n !}(Y) .
$$

By Lemma A.2, the Campbell-Hausdorff formula converges for all $X, Y \in \mathfrak{g}(\mathscr{L})$ :

$$
X * Y=X+Y+\sum_{k \geq 1} \frac{(-1)^{k}}{k+1} D_{k}(X, Y),
$$

where

$$
D_{k}(X, Y)=\sum_{l_{i}+m_{i}>0} \frac{\operatorname{ad}_{X}^{l_{1}}}{l_{1} !} \circ \frac{\operatorname{ad}_{Y}^{m_{1}}}{m_{1} !} \circ \ldots \circ \frac{\operatorname{ad}_{X}^{l_{k}}}{l_{k} !} \circ \frac{\operatorname{ad}_{Y}^{m_{k}}}{m_{k} !}(X) .
$$

We use the notation $\mathscr{G}(\mathscr{L})=\left\{e^{X} \mid X \in \mathfrak{g}(\mathscr{L})\right\}$; that is, $\mathscr{G}(\mathscr{L})$ is the same space as $\mathfrak{g}(\mathscr{L})$, but we denote its elements by $e^{X}$. The universal properties of the CampbellHausdorff formula (10) imply that $\mathscr{G}(\mathscr{L})$ endowed with the product $e^{X} e^{Y}=e^{X * Y}$ forms a group. Also, $A d$ gives an action of $\mathscr{G}(\mathscr{L})$ on $\mathscr{L}$ by graded Lie algebra automorphisms, which preserves the order:

- $\operatorname{Ad}\left(e^{X * Y}\right)=\operatorname{Ad}\left(e^{X} e^{Y}\right)=\operatorname{Ad}\left(e^{X}\right) \circ \operatorname{Ad}\left(e^{Y}\right)$,

- $\operatorname{Ad}\left(e^{X}\right)([U, V])=\left[\operatorname{Ad}\left(e^{X}\right) U, \operatorname{Ad}\left(e^{X}\right) V\right]$,

- $\mathcal{O}\left(\operatorname{Ad}\left(e^{X}\right)(U)\right)=\mathscr{O}(U)$,

for all $X, Y \in \mathfrak{g}(\mathscr{L})$ and all $U, V \in \mathscr{L}$.

For later use, we give the following straightforward estimates:

Lemma A.3. For all $X, Y, X^{\prime}, Y^{\prime} \in \mathfrak{g}(\mathscr{L})$ and $U \in \mathscr{L}$, we have

(1) $\mathcal{O}\left(X * Y-X^{\prime} * Y^{\prime}\right) \geq \mathcal{O}\left(X-X^{\prime}\right) \wedge \mathcal{O}\left(Y-Y^{\prime}\right)$ and

(2) $\mathcal{O}\left(\operatorname{Ad}\left(e^{X}\right) U-\operatorname{Ad}\left(e^{Y}\right) U\right) \geq \mathcal{O}(X-Y)$. 
Let $\gamma$ be an MC-element. Notice that $[\gamma, \gamma]=0$ implies that $d_{\gamma}:=\operatorname{ad}_{\gamma}$ is a differential on $\mathscr{L}^{\bullet}$. The fact that $\mathscr{F}_{k} \mathscr{L}$ are ideals implies that $\left(\mathscr{F}_{k} \mathscr{L}^{\bullet}, d_{\gamma}\right)$ are subcomplexes of $\left(\mathscr{L}^{\bullet}, d_{\gamma}\right)$. The induced differential on the consecutive complexes depends only on $\gamma$ modulo $\mathscr{F}_{1}$, and their cohomology groups are denoted

$$
H_{\gamma}^{n}\left(\mathscr{F}_{k} \mathscr{L}^{\bullet} / \mathscr{F}_{k+1} \mathscr{L}^{\bullet}\right) .
$$

$\operatorname{Ad}\left(e^{X}\right) \gamma$ is again an MC-element for $e^{X} \in \mathscr{G}(\mathscr{L})$, and we call $\gamma$ and $\operatorname{Ad}\left(e^{X}\right) \gamma$ gauge equivalent. The next Lemma gives a linear approximation of the action $\mathscr{G}(\mathscr{L})$ on MC-elements.

Lemma A.4. For $\gamma$ an MC-element and $e^{X} \in \mathscr{G}(\mathscr{L})$, we have

$$
\mathcal{O}\left(\operatorname{Ad}\left(e^{X}\right) \gamma-\gamma+d_{\gamma} X\right) \geq 2 \mathcal{O}(X) .
$$

We have the following criterion for gauge equivalence.

Theorem A.5. Let $\left(\mathscr{L}^{\bullet},[\cdot, \cdot]\right)$ be a GLA with a complete filtration $\mathscr{F}_{n} \mathscr{L}$, and let $\gamma, \gamma^{\prime}$ be two Maurer-Cartan elements. If $\mathcal{O}\left(\gamma-\gamma^{\prime}\right) \geq 1$, and iffor all $q \geq \mathcal{O}\left(\gamma-\gamma^{\prime}\right)$ we have

$$
H_{\gamma}^{1}\left(\mathscr{F}_{q} \mathscr{L}^{\bullet} / \mathscr{F}_{q+1} \mathscr{L}^{\bullet}\right)=0,
$$

then $\gamma$ and $\gamma^{\prime}$ are gauge equivalent; that is, there exists an element $e^{X} \in \mathscr{G}(\mathscr{L})$ such that $\gamma=\operatorname{Ad}\left(e^{X}\right) \gamma^{\prime}$.

Proof. Define $p:=\mathcal{O}\left(\gamma-\gamma^{\prime}\right)$. By hypothesis, for $q \geq p$, we can find homotopy operators

$$
h_{1}^{q}: \mathscr{F}_{q} \mathscr{L}^{1} \rightarrow \mathscr{F}_{q} \mathscr{L}^{0} \quad \text { and } \quad h_{2}^{q}: \mathscr{F}_{q} \mathscr{L}^{2} \rightarrow \mathscr{F}_{q} \mathscr{L}^{1}
$$

such that $h_{1}^{q}\left(\mathscr{F}_{q+1} \mathscr{L}^{1}\right) \subset \mathscr{F}_{q+1} \mathscr{L}^{0}, h_{2}^{q}\left(\mathscr{F}_{q+1} \mathscr{L}^{2}\right) \subset \mathscr{F}_{q+1} \mathscr{L}^{1}$ and

$$
\left(d_{\gamma} h_{1}^{q}+h_{2}^{q} d_{\gamma}-I d\right)\left(\mathscr{F}_{q} \mathscr{L}^{1}\right) \subset \mathscr{F}_{q+1} \mathscr{L}^{1} .
$$

We first prove an estimate. Let $q \geq p$, and let $\tilde{\gamma}$ be an MC-element such that $\mathcal{O}(\tilde{\gamma}-\gamma) \geq q$. Then for $\tilde{X}:=h_{1}^{q}(\tilde{\gamma}-\gamma)$, we claim that the following estimates hold:

$$
\mathcal{O}(\tilde{X}) \geq q, \quad \mathcal{O}\left(\operatorname{Ad}\left(e^{\tilde{X}}\right) \tilde{\gamma}-\gamma\right) \geq q+1 .
$$

The first follows by the properties of $h_{1}^{q}$, and to prove the second we compute:

$$
\begin{aligned}
\mathcal{O}\left(\operatorname{Ad}\left(e^{\tilde{X}}\right) \tilde{\gamma}-\gamma\right) & \geq \mathcal{O}\left(\operatorname{Ad}\left(e^{\tilde{X}}\right) \tilde{\gamma}-\tilde{\gamma}+d_{\tilde{\gamma}}(\tilde{X})\right) \wedge \mathcal{O}\left(\tilde{\gamma}-d_{\tilde{\gamma}}(\tilde{X})-\gamma\right) \\
& \geq 2 \mathcal{O}(\tilde{X}) \wedge \mathcal{O}([\gamma-\tilde{\gamma}, \tilde{X}]) \wedge \mathcal{O}\left(\tilde{\gamma}-\gamma-d_{\gamma}(\tilde{X})\right) \\
& \geq 2 q \wedge(\mathcal{O}(\gamma-\tilde{\gamma})+\mathcal{O}(\tilde{X})) \wedge \mathcal{O}\left(\tilde{\gamma}-\gamma-d_{\gamma}(\tilde{X})\right) \\
& \geq 2 q \wedge \mathcal{O}\left(\left(I d-d_{\gamma} h_{1}^{q}\right)(\tilde{\gamma}-\gamma)\right),
\end{aligned}
$$


where for the second inequality we use Lemma A.4. The last term can be evaluated as follows:

$$
\begin{aligned}
\mathcal{O}\left(\left(I d-d_{\gamma} h_{1}^{q}\right)(\tilde{\gamma}-\gamma)\right) & \geq \mathcal{O}\left(\left(I d-d_{\gamma} h_{1}^{q}-h_{2}^{q} d_{\gamma}\right)(\tilde{\gamma}-\gamma)\right) \wedge \mathcal{O}\left(h_{2}^{q}\left(d_{\gamma}(\tilde{\gamma}-\gamma)\right)\right) \\
& \geq(q+1) \wedge \mathcal{O}\left(h_{2}^{q}\left(d_{\gamma}(\tilde{\gamma}-\gamma)\right)\right) .
\end{aligned}
$$

Since $d_{\gamma}(\tilde{\gamma}-\gamma)=-\frac{1}{2}[\tilde{\gamma}-\gamma, \tilde{\gamma}-\gamma]$, we have $\mathcal{O}\left(d_{\gamma}(\tilde{\gamma}-\gamma)\right) \geq 2 q \geq q+1$, so

$$
\mathcal{O}\left(\left(I d-d_{\gamma} h_{1}^{q}\right)(\tilde{\gamma}-\gamma)\right) \geq q+1 \text {, }
$$

and this proves (11).

We construct a sequence of MC-elements $\left\{\gamma_{k}\right\}_{k \geq 0}$ and a sequence of group elements $\left\{e^{X_{k}}\right\}_{k \geq 1} \in \mathscr{G}(\mathscr{L})$ by the following recursive formulas:

$$
\begin{aligned}
\gamma_{0} & :=\gamma^{\prime}, \\
X_{k} & :=h_{1}^{p+k-1}\left(\gamma_{k-1}-\gamma\right) \quad \text { for } k \geq 1, \\
\gamma_{k} & :=\operatorname{Ad}\left(e^{X_{k}}\right) \gamma_{k-1} \quad \text { for } k \geq 1 .
\end{aligned}
$$

To show that these formulas do indeed give well-defined sequences, we have to check that $\gamma_{k-1}-\gamma \in \mathscr{F}_{p+k-1} \mathscr{L}^{1}$. This holds for $k=1$, and in general it follows by applying the estimate (11) inductively at each step $k \geq 1$ to $\tilde{\gamma}=\gamma_{k-1}$ and $q=p+k-1$, to obtain

$$
\mathcal{O}\left(X_{k}\right) \geq p+k-1, \quad \mathcal{O}\left(\gamma_{k}-\gamma\right) \geq p+k .
$$

Using Lemma A.3(1), we obtain

$$
\mathcal{O}\left(X_{k} * X_{k-1} \cdots * X_{1}-X_{k-1} \cdots * X_{1}\right) \geq \mathcal{O}\left(X_{k}\right) \geq p+k-1,
$$

and therefore by Lemma A.2, the product $X_{k} * X_{k-1} * \cdots * X_{1}$ converges to some element $X$. Applying Lemma A.3(1) $k$ times, we obtain

$$
\mathcal{O}\left(X_{k} * X_{k-1} \cdots * X_{1}\right) \geq \mathcal{O}\left(X_{k}\right) \wedge \mathcal{O}\left(X_{k-1}\right) \wedge \cdots \wedge \mathcal{O}\left(X_{1}\right) \geq 1,
$$

and thus $X \in \mathfrak{g}(\mathscr{L})$. On the other hand, we have

$$
\begin{aligned}
\mathcal{O}\left(\operatorname{Ad}\left(e^{X}\right) \gamma^{\prime}-\gamma\right) & \geq \mathcal{O}\left(\operatorname{Ad}\left(e^{X}\right) \gamma^{\prime}-\gamma_{k}\right) \wedge \mathcal{O}\left(\gamma_{k}-\gamma\right) \\
& \geq \mathcal{O}\left(\operatorname{Ad}\left(e^{X}\right) \gamma^{\prime}-\operatorname{Ad}\left(e^{X_{k} * \cdots * X_{1}}\right) \gamma^{\prime}\right) \wedge(p+k) \\
& \geq \mathcal{O}\left(X-X_{k} * \cdots * X_{1}\right) \wedge(p+k),
\end{aligned}
$$

where for the last estimate we used Lemma A.3(2). If we let $k \rightarrow \infty$, we obtain the conclusion: $\operatorname{Ad}\left(e^{X}\right) \gamma^{\prime}=\gamma$.

\section{Acknowledgment}

I would like to thank Marius Crainic for his very useful suggestions and comments. 


\section{References}

[Abad et al. 2010] C. A. Abad, M. Crainic, and B. Dherin, “Tensor products of representations up to homotopy", preprint, 2010. arXiv 1009.5852v1

[Bursztyn et al. 2009] H. Bursztyn, V. Dolgushev, and S. Waldmann, "Morita equivalence and characteristic classes of star products", preprint, 2009. arXiv 0909.4259

[Conn 1985] J. F. Conn, "Normal forms for smooth Poisson structures", Ann. of Math. (2) 121:3 (1985), 565-593. MR 86m:58050 Zbl 0592.58025

[Crainic 2003] M. Crainic, "Differentiable and algebroid cohomology, Van Est isomorphisms, and characteristic classes", Comment. Math. Helv. 78:4 (2003), 681-721. MR 2004m:58034 Zbl 1041. 58007

[Crainic and Fernandes 2003] M. Crainic and R. L. Fernandes, "Integrability of Lie brackets", Ann. of Math. (2) 157:2 (2003), 575-620. MR 2004h:58027 Zbl 1037.22003

[Crainic and Mărcuţ 2010] M. Crainic and I. T. Mărcuţ, "A normal form theorem around symplectic leaves", preprint, 2010. arXiv 1009.2090v1

[Duistermaat and Kolk 2000] J. J. Duistermaat and J. A. C. Kolk, Lie groups, Springer, Berlin, 2000. MR 2001j:22008 Zbl 0955.22001

[Ginzburg and Lu 1992] V. L. Ginzburg and J.-H. Lu, "Poisson cohomology of Morita-equivalent Poisson manifolds", Int. Math. Res. Notices 1992:10 (1992), 199-205. MR 93k:58091 Zbl 0783. 58026

[Haefliger 1979] A. Haefliger, "Differentiable cohomology", pp. 19-70 in Differential topology (Varenna, 1976), edited by V. Villani, Liguori, Naples, 1979. MR 83k:17014 Zbl 0467.57007

[Hochschild and Serre 1953] G. Hochschild and J.-P. Serre, "Cohomology of Lie algebras", Ann. of Math. (2) 57:3 (1953), 591-603. MR 14,943c Zbl 0053.01402

[Itskov et al. 1998] V. Itskov, M. Karasev, and Y. Vorobjev, "Infinitesimal Poisson cohomology", pp. 327-360 in Coherent transform, quantization, and Poisson geometry, edited by M. V. Karasev, Amer. Math. Soc. Transl. Ser. 2 187, Amer. Math. Soc., Providence, RI, 1998. MR 2001k:58031 Zbl 0922.58028

[Mackenzie 1987] K. Mackenzie, Lie groupoids and Lie algebroids in differential geometry, London Mathematical Society Lecture Note Series 124, Cambridge University Press, Cambridge, 1987. MR 89g:58225 Zbl 0683.53029

[Moerdijk and Mrčun 2003] I. Moerdijk and J. Mrčun, Introduction to foliations and Lie groupoids, Cambridge Studies in Advanced Mathematics 91, Cambridge University Press, Cambridge, 2003. MR 2005c:58039 Zbl 1029.58012

[Moerdijk and Reyes 1991] I. Moerdijk and G. E. Reyes, Models for smooth infinitesimal analysis, Springer, New York, 1991. MR 91m:18017 Zbl 0715.18001

[Vaisman 1994] I. Vaisman, Lectures on the geometry of Poisson manifolds, Progress in Mathematics 118, Birkhäuser, Basel, 1994. MR 95h:58057 Zbl 0810.53019

[Vorobjev 2001] Y. Vorobjev, "Coupling tensors and Poisson geometry near a single symplectic leaf", pp. 249-274 in Lie algebroids and related topics in differential geometry (Warsaw, 2000), edited by J. Kubarski et al., Banach Center Publ. 54, Polish Acad. Sci. Inst. Math., Warsaw, 2001. MR 2003d:53145 Zbl 1007.53062

[Weinstein 1983] A. Weinstein, "The local structure of Poisson manifolds", J. Differential Geom. 18:3 (1983), 523-557. MR 86i:58059 Zbl 0524.58011

Received November 27, 2010. Revised June 29, 2011. 
EQUIVALENCE OF POISSON STRUCTURES AROUND POISSON SUBMANIFOLDS 461

IOAN MĂRCUT,

DEPARTMENT OF MATHEMATICS

UTRECHT UNIVERSITY

3508 TA UTRECHT

THE NETHERLANDS

I.T.Marcut@uu.nl 


\title{
PACIFIC JOURNAL OF MATHEMATICS
}

\author{
http://pacificmath.org \\ Founded in 1951 by \\ E. F. Beckenbach (1906-1982) and F. Wolf (1904-1989)
}

\section{EDITORS}

V. S. Varadarajan (Managing Editor)

Department of Mathematics

University of California

Los Angeles, CA 90095-1555

pacific@math.ucla.edu

Vyjayanthi Chari

Department of Mathematics

University of California

Riverside, CA 92521-0135

chari@math.ucr.edu

\section{Robert Finn}

Department of Mathematics Stanford University

Stanford, CA 94305-2125

finn@math.stanford.edu

Kefeng Liu

Department of Mathematics

University of California

Los Angeles, CA 90095-1555

liu@math.ucla.edu
Darren Long

Department of Mathematics

University of California

Santa Barbara, CA 93106-3080

long@math.ucsb.edu

Jiang-Hua Lu

Department of Mathematics

The University of Hong Kong

Pokfulam Rd., Hong Kong jhlu@maths.hku.hk

Alexander Merkurjev

Department of Mathematics

University of California

Los Angeles, CA 90095-1555

merkurev@math.ucla.edu
Sorin Popa

Department of Mathematics University of California

Los Angeles, CA 90095-1555 popa@math.ucla.edu

Jie Qing

Department of Mathematics

University of California

Santa Cruz, CA 95064

qing@cats.ucsc.edu

Jonathan Rogawski

Department of Mathematics

University of California

Los Angeles, CA 90095-1555

jonr@math.ucla.edu

\section{PRODUCTION}

pacific@math.berkeley.edu

\section{SUPPORTING INSTITUTIONS}

ACADEMIA SINICA, TAIPEI

CALIFORNIA INST. OF TECHNOLOGY INST. DE MATEMÁTICA PURA E APLICADA KEIO UNIVERSITY

MATH. SCIENCES RESEARCH INSTITUTE NEW MEXICO STATE UNIV.

OREGON STATE UNIV.

\author{
STANFORD UNIVERSITY \\ UNIV. OF BRITISH COLUMBIA \\ UNIV. OF CALIFORNIA, BERKELEY \\ UNIV. OF CALIFORNIA, DAVIS \\ UNIV. OF CALIFORNIA, LOS ANGELES \\ UNIV. OF CALIFORNIA, RIVERSIDE \\ UNIV. OF CALIFORNIA, SAN DIEGO \\ UNIV. OF CALIF., SANTA BARBARA
}

\author{
UNIV. OF CALIF., SANTA CRUZ \\ UNIV. OF MONTANA \\ UNIV. OF OREGON \\ UNIV. OF SOUTHERN CALIFORNIA \\ UNIV. OF UTAH \\ UNIV. OF WASHINGTON \\ WASHINGTON STATE UNIVERSITY
}

These supporting institutions contribute to the cost of publication of this Journal, but they are not owners or publishers and have no responsibility for its contents or policies.

See inside back cover or pacificmath.org for submission instructions.

The subscription price for 2012 is US \$420/year for the electronic version, and \$485/year for print and electronic.

Subscriptions, requests for back issues from the last three years and changes of subscribers address should be sent to Pacific Journal of Mathematics, P.O. Box 4163, Berkeley, CA 94704-0163, U.S.A. Prior back issues are obtainable from Periodicals Service Company, 11 Main Street, Germantown, NY 12526-5635. The Pacific Journal of Mathematics is indexed by Mathematical Reviews, Zentralblatt MATH, PASCAL CNRS Index, Referativnyi Zhurnal, Current Mathematical Publications and the Science Citation Index.

The Pacific Journal of Mathematics (ISSN 0030-8730) at the University of California, c/o Department of Mathematics, 969 Evans Hall, Berkeley, CA 94720-3840, is published monthly except July and August. Periodical rate postage paid at Berkeley, CA 94704, and additional mailing offices. POSTMASTER: send address changes to Pacific Journal of Mathematics, P.O. Box 4163, Berkeley, CA 94704-0163.

PJM peer review and production are managed by EditFLOW ${ }^{\mathrm{TM}}$ from Mathematical Sciences Publishers.

PUBLISHED BY PACIFIC JOURNAL OF MATHEMATICS

at the University of California, Berkeley 94720-3840

A NON-PROFIT CORPORATION

Typeset in LATEX

Copyright $(02012$ by Pacific Journal of Mathematics 


\section{PACIFIC JOURNAL OF MATHEMATICS}

Volume $255 \quad$ No. $2 \quad$ February 2012

On the local Langlands correspondences of DeBacker-Reeder and

Reeder for $\operatorname{GL}(\ell, F)$, where $\ell$ is prime

MOSHE ADRIAN

$R$-groups and parameters

281

DUBRAVKA BAN and DAVID GOLDBERG

Finite-volume complex-hyperbolic surfaces, their toroidal

305

compactifications, and geometric applications

LUCA FABRIZIO Di CERBO

Character analogues of Ramanujan-type integrals involving the Riemann 317 $\Xi$-function

ATUl DiXIT

Spectral theory for linear relations via linear operators

DANA GHEORGHE and FLORIAN-HORIA VASILESCU

Homogeneous links and the Seifert matrix

PEDRO M. GonZÁlez MANCHÓN

Quantum affine algebras, canonical bases, and $q$-deformation of arithmetical functions

HENRY H. KIM and KYU-HWAN LEE

Dirichlet-Ford domains and arithmetic reflection groups

GRANT S. LAKELAND

Formal equivalence of Poisson structures around Poisson submanifolds

IOAN MĂRCUT,

A regularity theorem for graphic spacelike mean curvature flows

BENJAMIN STUART THORPE

Analogues of level- $N$ Eisenstein series 